Abanico Veterinario. Enero-Diciembre 2021; 11:1-12. http://dx.doi.org/10.21929/abavet2021.21

Nota Corta. Recibido: 26/01/2021. Aceptado: 20/04/2021. Publicado: 10/05/2021. Clave: e2021-8.

\title{
Seroprevalencia de Mycobacterium avium subespecie paratuberculosis en bovinos sospechosos sacrificados en rastro de Sonora, México
}

Seroprevalence of Mycobacterium avium subspecie paratuberculosis in suspect cattle slaughtered at slaughterhouse from Sonora, Mexico

\section{Reyna Osuna-Chávez ${ }^{1 \mathrm{ID}}$, Guadalupe López-Robles ${ }^{11 \mathrm{D}}$, Hernán Celaya-Michel ${ }^{11 \mathrm{D}}$, Jesús Sosa-Castañeda ${ }^{11 \mathrm{D}}$, Manuel Nieblas-López ${ }^{11 \mathrm{D}}$, Cristina Ibarra-Zazueta ${ }^{1 \mathrm{ID}^{*}}$}

1Departamento de Agricultura y Ganadería, Universidad de Sonora. México. *Autor responsable y de correspondencia: Ibarra-Zazueta Cristina. Departamento de Agricultura y Ganadería, Universidad de Sonora. Carretera Bahía de Kino Km 21. Apartado postal 305. Hermosillo, Sonora, México. reyna.osuna@unison.mx, guadalupe.lopez@unison.mx, hernan.celaya@unison.mx, jesus.sosa@unison.mx,manuel.nieblas@unison.mx,cristina.ibarra@unison.mx

\section{RESUMEN}

La paratuberculosis en una enfermedad infecto-contagiosa causada por la bacteria Mycobacterium avium subespecie paratuberculosis (MAP), y afecta al aparato digestivo de los rumiantes. MAP es de distribución mundial, sin embargo, no hay publicaciones de la seroprevalencia en bovinos de esta enfermedad en el estado de Sonora. Por lo anterior, el objetivo de este trabajo fue detectar la seropositividad a MAP en ganado bovino de Sonora, así como conocer su prevalencia aparente en una población de bovinos adultos con emaciación y caquexia. Se analizaron 385 muestras de suero de bovinos, 370 de hembras y 15 de machos, mayores de 3 años, nacidos en la entidad con signos de emaciación y caquexia. Se recopilaron datos sobre edad, raza, fin zootécnico y municipio de origen. La finalidad zootécnica de los animales fue en su mayoría bovinos de doble propósito con $84.93 \%$ (370/385). Las muestras procesaron mediante la prueba comercial ELISA. Los resultados muestran una seroprevalencia aparente de $2.08 \%(8 / 385)$ y los municipios que presentaron animales seropositivos fueron Hermosillo con 3 muestras, así como, Guaymas, Huachinera, Ures, Villa Pesqueira y Fronteras, con una muestra cada uno. El presente estudio demuestra por primera vez la seropositividad a Mycobacterium avium subespecie paratuberculosis en ganado bovino de Sonora. Además de una baja seroprevalencia aparente en la población de bovinos muestreada. La presencia de MAP en Sonora, la cual podría ser considerada como una enfermedad olvidada, pone en evidencia la necesidad de conocer su posible rol en la salud pública de México.

Palabras clave: Seroprevalencia, Mycobacterium avium subspecie paratuberculosis, Sonora.

\section{ABSTRACT}

Paratuberculosis is an infectious-contagious disease caused by the bacterium Mycobacterium avium subspecie paratuberculosis (MAP), which affects the digestive tract of ruminants. MAP is distributed worldwide; however, there are no publications on the seroprevalence of this disease in cattle in the state of Sonora. Therefore, the objective of this study was to detect MAP seropositivity in cattle in Sonora, as well as to know its apparent prevalence in a population of adult cattle with emaciation and cachexia. Three hundred and eighty-five serum samples were analyzed from 370 female and 15 male cattle, older than 3 years, born in the state with signs of emaciation and cachexia. Data were collected on age, breed, zootechnical purpose and municipality of origin. The zootechnical purpose of the animals was mostly dual purpose cattle with $84.93 \%$ (370/385). The samples were processed using the commercial ELISA test. The results show an apparent seroprevalence of $2.08 \%(8 / 385)$ and the municipalities that presented seropositive animals were Hermosillo with 3 samples, as well as Guaymas, Huachinera, Ures, Villa 
Pesqueira and Fronteras, with one sample each. The present study demonstrates for the first time seropositivity to Mycobacterium avium subspecies paratuberculosis in cattle in Sonora. In addition to a low apparent seroprevalence in the cattle population sampled. The presence of MAP in Sonora, which could be considered a neglected disease, highlights the need to know its possible role in public health in Mexico.

Keywords: Seroprevalence, Mycobacterium avium subspecie paratuberculosis, Sonora.

\section{INTRODUCCIÓN}

La paratuberculosis (PTBC) o enfermedad de Johne, es una enfermedad crónica y contagiosa causada por la bacteria Mycobacterium avium, subespecie paratuberculosis (MAP). En el acuerdo mediante el cual se dan a conocer en los Estados Unidos Mexicanos las enfermedades y plagas exóticas y endémicas de notificación obligatoria de los animales terrestres y acuáticos del Diario Oficial de la Federación, publicado en noviembre del 2018, la PTBC se encuentra catalogada dentro del grupo 3 de enfermedades y plagas, las cuales están constituidas por aquellas que se encuentran en territorio nacional, consideradas como endémicas, que representan un menor riesgo desde el punto de vista epidemiológico, económico, de salud púbica y para el comercio nacional e internacional; ya que pueden ser controlables y son de notificación mensual obligatoria (DOF, 2018). La infección por MAP, afecta a bovinos, ovinos y caprinos; principalmente de producción lechera (Retamal et al., 2011; OIE, 2020). Causa lesiones granulomatosas en aparato digestivo y se caracteriza por causar enteritis granulomatosa proliferativa y linfadenitis con infiltración mononuclear, lo que provoca síndrome de mala absorción a los animales infectados, afectando el desempeño productivo de los animales (Rathanaiah et al., 2017). Uno de los principales mecanismos de infección se da en los terneros, cuando se infectan durante la lactancia por medio de infestación oral-fecal; aunque no se descarta que pudieran contagiarse en la etapa de estabulación.

El periodo de incubación de MAP oscila entre 4 meses a 15 años, y los signos clínicos pueden aparecer entre los dos y los cinco años (Eisenber et al., 2012). Debido a las similitudes fisiopatológicas entre la PTBC en rumiantes y la enfermedad de Crohn en humanos, se ha sugerido que se pudiera tratar de una enfermedad zoonótica (Naser et al., 2004); sin embargo, hasta el momento no se ha podido demostrar la relación directa entre la infección por MAP y la enfermedad de Crohn en humanos (Chaubey et al., 2017). Se ha realizado PCR del gen IS900 y la prevalencia real de MAP en pacientes afectados por enfermedad de Crohn y la enfermedad intestinal no inflamatoria fue de 47 y $13 \%$ respectivamente; sólo el $7 \%$ de los pacientes con la enfermedad de Crohn fueron moderadamente positivos a la tinción Zielh Nelseen. En los hallazgos en histopatología se observó enteritis granulomatosa (83 y $90 \%$ ), enteritis linfoplasmocítica (17 y $14 \%$ ), edema y linfagiectasia (67 y $96 \%$ ) y vasculitis (20 y $73 \%$ ), para enfermedad de Crohn y MAP, respectivamente (Zarei et al., 2019). 
El diagnóstico de la PTB puede ser por medio de pruebas de ELISA, PCR, aislamiento bacteriano, fijación de complemento, inmunodifusión en gel de agar; así como tinción de Ziehl Neelsen en muestras de histopatología y heces; también se puede realizar por medio de la detección de inmunidad mediada por células (prueba de piel ano caudal); sin embargo presenta reacción cruzada con Mycobacterium bovis (Whittington et al., 2019). La PTBC se encuentra distribuida a nivel mundial, en una encuesta realizada a 48 países se encontró que la PTBC es muy común, en aproximadamente la mitad de estos países, más del $20 \%$ de los rebaños estaban infectados por MAP (Whittington et al., 2019). Se ha demostrado que sólo Suecia, Noruega y algunos estados de Australia se encuentran libres de la enfermedad, porque en estos lugares realizan informes válidos y medidas de control de MAP, y porque el objetivo es la erradicación de la enfermedad (Collins et al., 2010; Eslami et al 2019; Whittington et al., 2019). La PTBC presenta una prevalencia en los bovinos a nivel mundial entre 7 a $60 \%$, siendo menor en ganado de carne que en ganado lechero; además, se ha calculado que en ovinos es del $16 \%$ en América Latina y el Caribe (Fernández et al., 2014).

En México, la información que se tiene sobre seroprevalencia de MAP en bovinos es escasa, los estudios realizados han sido principalmente en ganado ovino y caprino. En ovinos de San Luis Potosí se detectó una seropositividad de 9.48\% (Morón et al., 2013); mientras que en Jalisco, Guanajuato y el estado de México fue de $44.6 \%$ (Jaimes et al., 2008), en Nayarit 5.16\% (Mejía et al., 2017), en Baja California 7.8\% (Correa et al., 2013). En Sonora se reportó una seroprevalencia general para ovinos del $7.48 \%$ y al analizarlo por regiones, la mayor seropositividad fue observada en el sur del estado con $11.10 \%$ (Morales et al., 2020). En otros estudios de México se ha reportado la presencia de MAP en caprinos, en Veracruz se detectó una seropositividad del 0.6\% (Martínez et al., 2012), en la parte central de México de 8.29-9.67\% (Favila et al., 2010) y en Puebla se encontró una seroprevalencia del 48.1\% (Gallaga et al., 2017). En el estudio realizado por Morales et al. (2020), se analizaron los factores de riesgo para la presentación de seropositividad a MAP en ovinos de Sonora y se encontró que el riesgo fue mayor cuando los animales nacieron fuera de la explotación y también en explotaciones con más de 300 animales. Aunque en bovinos la información sobre MAP es limitada, se ha observado que existe una seroprevalencia del 8.29-9.67\% en el área central de México (Favila et al., 2010).

En un estudio realizado por Milián et al. (2015), donde analizaron la seroprevalencia de MAP en bovinos de diferentes estados de México, se detectó una seroprevalencia de $5 \%$ para el país; y los estados que mostraron mayor seroprevalencia fueron Jalisco y Guanajuato, con $11 \%$ y $10 \%$, respectivamente. En este mismo estudio también se incluyeron los estados de Chihuahua y Sinaloa, los cuales colindan con Sonora y se detectó una seroprevalencia de MAP de $2 \%$ y $6 \%$, respectivamente. Lamentablemente no existe información disponible sobre la situación actual de MAP en bovinos de Sonora, pero la presencia de la enfermedad en ovinos de este estado; así como en ovinos y bovinos de estados colindantes, sugieren que la enfermedad podría estar circulando en el ganado bovino del estado de Sonora. 
El objetivo de este trabajo fue detectar la presencia de seropositividad a Mycobacterium avium subespecie paratuberculosis en ganado bovino de diferentes municipios de Sonora, así como conocer cuál sería su prevalencia aparente en una población de bovinos clínicamente sospechosos (con emaciación y caquexia) y mayores de 3 años.

\section{MATERIAL Y MÉTODOS}

Diseño del estudio, tamaño de la muestra y colecta.

Se realizó un estudio epidemiológico observacional transversal entre mayo de 2018 y mayo de 2019; el tamaño de la muestra $(n=385)$, se determinó con la metodología descrita por Segura y Hondhold (2015); para esto se consideró la población total de 1,728,001 bovinos del estado de Sonora: 1,709,366 de producción de carne y 18,635 de leche (SIAP, 2018), con un nivel de confianza del $95 \%$, un error estimado del $5 \%$ y una prevalencia esperada del $5 \%$. Se realizó un muestreo no probabilístico por conveniencia. Los animales se seleccionaron de un rastro TIF de la ciudad de Hermosillo, que recibe bovinos de todo el estado de Sonora. Como criterio de inclusión se consideró que los animales tuvieran arete SINIIGA y marca de herrar que los identificara como procedentes de Sonora, que fueran mayores de 3 años y que mostraran signos de emaciación y caquexia. El criterio de exclusión fue que no se colectarían muestras de animales menores de 3 años y con condición corporal de 3-5. Además, se recopilaron datos sobre la finalidad zootécnica de los animales, edad, sexo y municipio al que pertenecían. Para clasificar las muestras de acuerdo con la situación geográfica de donde provenían los animales, se utilizó el Sistema Estatal de Información para el Ordenamiento Territorial del Estado de Sonora (SEIOT), que agrupa a diferentes municipios en unidades territoriales, que se muestran en la figura 1 (SEIOT, 2020).

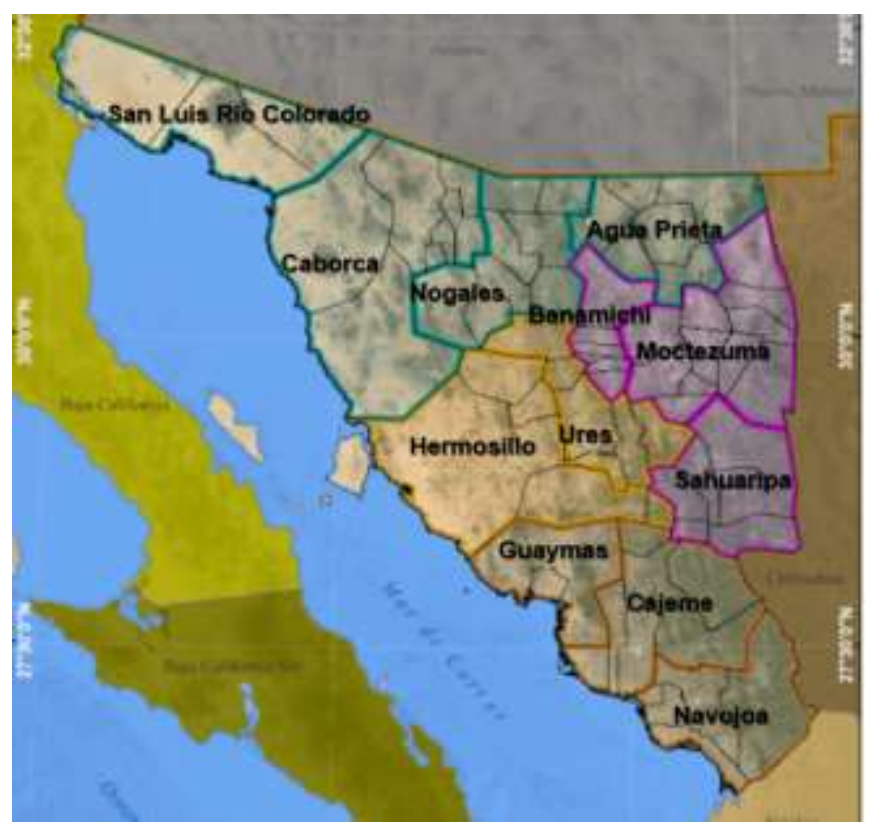

Figura 1. Unidades Territoriales Estado de Sonora muestreados (SEIOT, 2020) 
Obtención y procesamiento de las muestras.

Se colectaron muestras de sangre de la vena yugular de cada animal en tubos de $10 \mathrm{~mL}$ sin anticoagulante (Vaccutainer®); el procedimiento se realizó de acuerdo con la NOM062-ZOO-1999. La detección de anticuerpos anti-MAP se realizó a través de la técnica de ELISA indirecta (Jark et al.,1997), con un estuche comercial (IDEXX Laboratories, Inc., EUA), y se realizaron de acuerdo con las instrucciones del fabricante. La medición se realizó en un lector de ELISA (Stat Fax ®4700), a una absorbancia de $450 \mathrm{~nm}$. Se consideraron positivas las muestras con un $\mathrm{S} / \mathrm{P}>55 \%$, dudosas de 45 a $55 \%$ y negativo $<45 \%$.

Análisis de datos. Para conocer la seroprevalencia aparente de MAP en la población de bovinos analizada (adultos con emaciación y caquexia), se consideraron los animales positivos a MAP por la prueba de ELISA y se dividieron entre el número total de la población muestreada.

\section{RESULTADOS Y DISCUSIÓN}

Se muestrearon $96.10 \%$ (370/385) hembras y $3.90 \%$ (15/385) machos. Se encontró que el $3.90 \%(15 / 385)$ de los animales muestreados fueron bovinos productores de carne, el $84.93 \%$ (327/385) doble propósito (producción de carne y leche) y el $11.17 \%$ (43/385); la finalidad zootécnica fue la producción de leche. El promedio de edad de los animales seropositivos a MAP fue de $7.5 \pm 2$ años, lo cual, de acuerdo con la literatura e infección por MAP, usualmente ocurre en los primeros meses de vida de los animales, en la etapa de lactancia por leche contaminada con heces, pero los signos clínicos se observan después de un largo periodo de incubación y generalmente aparecen entre los dos y los cinco años (Craven et al., 2000). Los animales muestreados en este estudio fueron principalmente bovinos de desecho; además presentaron los signos característicos de PTBC que son emaciación y caquexia. Aunque el diagnóstico presuntivo de la enfermedad puede realizarse con base en los signos clínicos y los hallazgos a la necropsia, existen otras enfermedades que tienen las mismas manifestaciones clínicas; debido a ésto, se requiere la confirmación de laboratorio y pruebas serológicas (Bustamante et al., 2011).

Se obtuvieron muestras serológicas de ganado bovino de 55 de los 72 municipios del estado de Sonora (tabla 1), lo que corresponde a un $76.39 \%$ del territorio estatal; dichos municipios se encuentran en la parte centro norte del estado (fig. 1).

El municipio con mayor número de muestras fue Hermosillo con un $45.45 \%$ (175/385). En el análisis serológico para la detección de anticuerpos anti-MAP, se observó que los municipios donde se detectaron muestras dudosas fueron: Hermosillo $(n=3)$, Guaymas $(n=1)$, Huachinera $(n=1)$, Ures $(n=1)$, Villa Pesqueira $(n=1)$ y Fronteras $(n=1)$ (fig. 2). 
Tabla 1. Municipios del estado de Sonora evaluados en el estudio

\begin{tabular}{|c|c|c|}
\hline Unidad Territorial Básica & $\begin{array}{l}\text { No. de } \\
\text { Animales }\end{array}$ & Municipios que se incluyen \\
\hline Hermosillo & 175 & Hermosillo, San Miguel de Horcasitas, La Colorada, San Javier, Carbo \\
\hline Guaymas & 13 & Guaymas, Empalme \\
\hline Cajeme & 12 & Cajeme, Suaqui Grande, Rosario, Quiriego, San Ignacio Río Muerto, Bácum \\
\hline San Luis Río Colorado & 2 & San Luis Rio Colorado, Puerto Peñasco, Gral. Plutarco Elías Calles \\
\hline Caborca & 16 & Caborca, Pitiquito, Saric, Áltar, Tubutama, Átil, Oquitoa \\
\hline Nogales & 23 & $\begin{array}{c}\text { Nogales, Santa Cruz, Ímuris, Magdalena, Santa Ana, Benjamín Hill, Trincheras, } \\
\text { Cucúrpe }\end{array}$ \\
\hline Agua Prieta & 11 & Agua Prieta, Fronteras, Nacozari de García, Bacoachi, Cananea, Naco \\
\hline Moctezuma & 49 & $\begin{array}{l}\text { Moctezuma, Bacerac, Huachinera, Nacori Chico, Bacadehuachi, Huasabas, } \\
\text { Granados, Divisaderos, Cumpas, Bavispe, Villa Hidalgo, Tepache }\end{array}$ \\
\hline Banámichi & 8 & Arizpe, Banamichi, Huepac, Aconchi, Baviacora, San Felipe de Jesús \\
\hline Sahuaripa & 24 & Sahuaripa, Arivechi, Yecora, Bacanora, Soyopa, Onavas \\
\hline Ures & 49 & Ures, Opodepe, Rayón, Villa Pesqueira, Mazatán, San Pedro de la Cueva \\
\hline Navojoa & 3 & Álamos, Etchojoa, Navojoa, Benito Juárez, Huatabampo \\
\hline
\end{tabular}




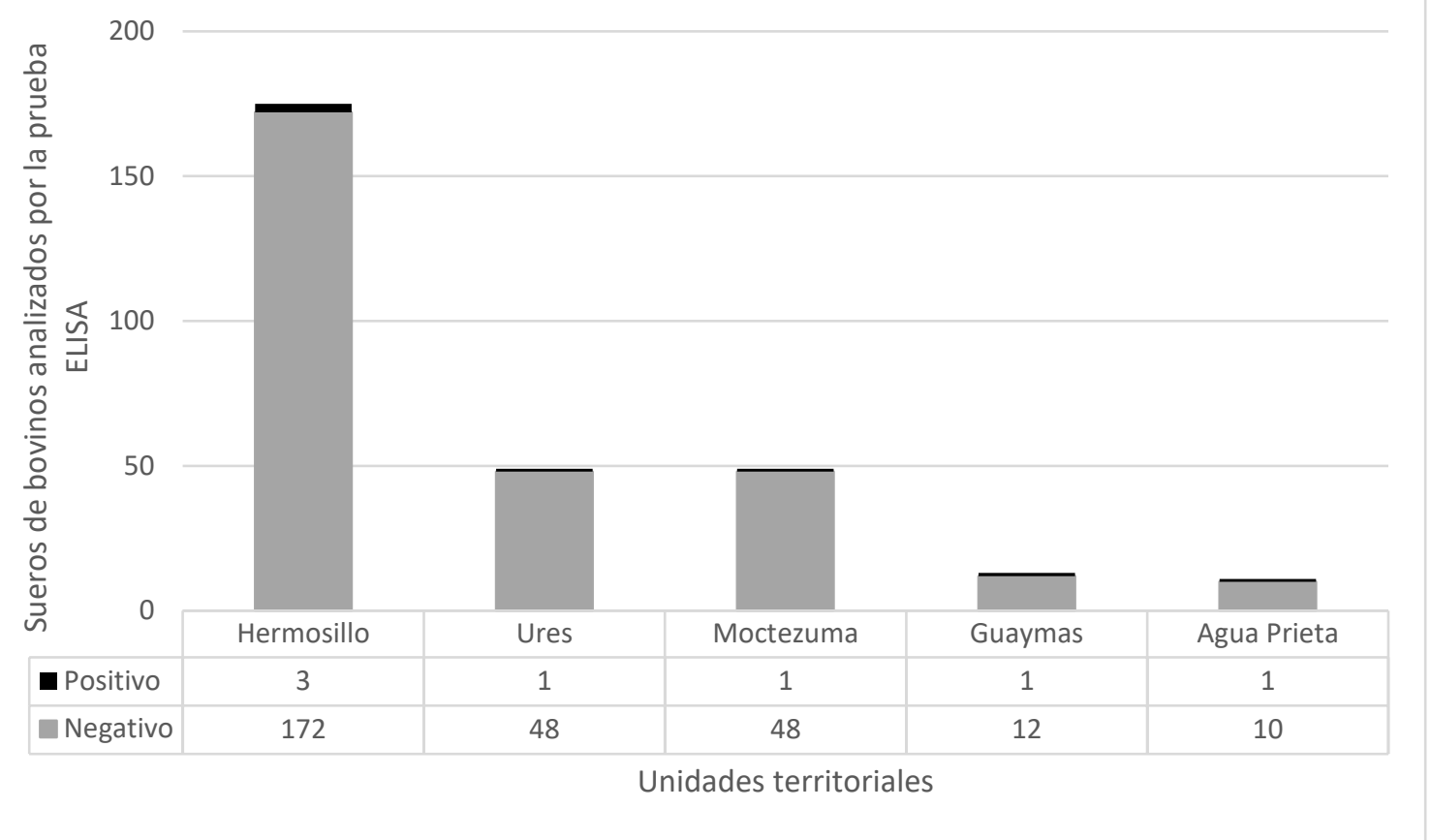

Figura 2. Unidades territoriales con muestras positivas a MAP

Existen diversos estudios de seroprevalecia de la enfermedad, en donde a las muestras que presentan resultados dudosos se les considera positivas (Velez et al., 2016; Jaramillo et al., 2017). La seroprevalencia aparente estimada para el estado fue de $2.08 \%$ (8/385); estos resultados coinciden con lo detectado en Chihuahua por Milián et al. (2015). Es sugestivo observar que, aunque Sonora también colinda con Sinaloa y Baja California, donde se detectaron seroprevalencias más altas (Milián et al., 2015), la introducción de ganado al estado se encuentra prohibida por su elevado estatus sanitario; esto es debido a que Sonora se encuentra en etapa de erradicación de Mycobacterium bovis (Tuberculosis bovina). Dentro de las pruebas oficiales para el diagnóstico de tuberculosis bovina en México, se utiliza la prueba de tuberculinización; las cuales se consideran la prueba de pliegue ano caudal, cervical comparada y cervical simple. Esta prueba es altamente sensible y presenta baja especificidad, y la prueba de pliegue ano caudal es ideal para identificar poblaciones reactoras a las micobacterias; por lo que para discriminar a quien pudiera corresponder esta reacción se utiliza la doble cervical comparada (NOM-031-ZOO-1995).

Existen similitudes inmunogénicas de MAP con Mycobacterium bovis, y dado a que se hizo un barrido con la prueba de tuberculina mediante el Plan Estratégico conjunto entre Estados Unidos - México para la colaboración en Tuberculosis bovina 2013-2018, y a que el estado de Sonora tiene avance como acreditado modificado avanzado para el USDA (Departamento de Agricultura de los Estados Unidos); la estrategia estatal se basa en la eliminación de reactores y despoblación de hatos positivos a pruebas de histopatología y aislamiento bacteriano (SENASICA, 2021). Esto ha permitido un gran 
avance en la campaña de tuberculosis bovina y de manera indirecta minimizar la presencia de MAP en los bovinos de la entidad; sin embargo, existe la evidencia de seropositividad para MAP en ovinos del estado (Morales et al., 2020); esto incrementa la posibilidad de haya la presencia de anticuerpos circulantes de MAP en la entidad y que en algún momento los bovinos tengan contacto con la enfermedad.

\section{CONCLUSIONES}

Los resultados obtenidos en el presente estudio demuestran por primera vez la seropositividad a Mycobacterium avium subespecie paratuberculosis en ganado bovino de Sonora. Además, se observó una baja seroprevalencia aparente en la población de bovinos muestreada. Sin embargo, para tener información más precisa sobre el comportamiento epidemiológico de este agente etiológico en la población de ganado bovino de Sonora, es necesario que a futuro se realicen más análisis en animales productivamente activos dentro de las unidades de producción. Por otro lado, la presencia de MAP en Sonora, la cual es una enfermedad olvidada, pone en evidencia la necesidad de conocer su posible rol en la salud pública de México.

\section{LITERATURA CITADA}

BUSTAMANTE VJ, Aguilar OJ, Ortíz MM, Bustamante L.J. 2011. Mycobacterium avium subespecie paratuberculosis en bovinos lecheros de la zona de Lima detectado mediante tres pruebas diagnósticas. Revista de investigaciones veterinarias Perú. 22 (4): 394 402. ISSN: 1609-9117. http://www.scielo.org.pe/pdf/rivep/v22n4/a14v22n4.pdf

CHAUBEY KK, Singh SV, Gupta S, Singh M, Sohal J S, Kumar N, Singh MK, Bhatia AK, Dhama K. 2017. Mycobacterium avium subespecie paratuberculosis - an important food borne pathogen of high public health significance with special reference to India: an update. Veterinary Quarterly. 37: 282 - 299.

https://doi.org/10.1080/01652176.2017.1397301

COLLINS MT, Eggleston V. Manning JB. 2010. Successful control of Johne's disease in nine dairy herds: Results of a six-year field trial. Journal Dairy Science. 93: 1638-1643. http://doi.org/10.3168/jds.2009-2664

CORREA MM, Medina BG, Rentería ET, Monge NF, González VV, López VG. 2013. Caracterización molecular de Mycobacterium avium subespecie paratuberculosis en bovinos y ovinos de Mexicali, Baja California, México. Revista mexicana de ciencias pecuarias. $\quad 4(4): \quad 489-500$.

ISSN

2448-6698.

http://www.scielo.org.mx/pdf/rmcp/v4n4/v4n4a6.pdf 
CRAVEN JA, Morgan IR. Epidemiology and Pathogenesis of Paratuberculosis in cattle. A literature survey prepared for animal health Australia. 2000. www.animalhealthaustralia.com.au/wp-content/uploads/2011/04/Epidemology-andPathogenesis-of-Johne\%E2\%80\%99s-Diseasein-Cattle.pdf

DOF. Diario Oficial de la Federación. 2018. ACUERDO mediante el cual se dan a conocer en los Estados Unidos Mexicanos las enfermedades y plagas exóticas y endémicas de notificación obligatoria de los animales terrestres y acuáticos. 29-11-2018. Secretaría de Agricultura, Ganadería, Desarrollo Rural, Pesca y Alimentación. México. https://dof.gob.mx/nota_detalle.php?codigo=5545304\&fecha=29/11/2018

EISENBERG SW, Nielen M, Koets AP. 2012. Within-farm transmission of bovine paratuberculosis: recent developments. Vet $Q$ 32(1):31-5. http://doi.org/10.1080/01652176.2012.659870

ESLAMI M, Shafiei M, Ghasemian A, Valizadeh S, Al-Marzoqi AH, Shokouhi Mostafavi SK, Nojoomi F, Mirforughi SA. 2019. Mycobacterium avium paratuberculosis and Mycobacterium avium complex and related subspecies as causative agents of zoonotic and occupational diseases. J Cell Physiol. 234(8):12415-12421. http://doi.org/10.1002/jcp.28076

FAVILA HL, Chávez GG, Carrillo CEM, Hernández CR. 2010. Mycobacterium avium subsp. paratuberculosis detection in individual and bulk tank milk samples from bovine herds and caprine flocks. Foodborne pathogens and disease. 7(4): 351 - 355. http://doi.org/10.1089/fpd.2009.0374

FERNÁNDEZ SJA, Correa VNM, Ramírez NF. 2014. Systematic review of the prevalence of paratuberculosis in cattle, sheep, and goats in Latin America and the Caribbean. Trop. Anim. Health Prod. 46:1321 - 1340. http://doi.org/10.1007/s11250-014-0656-8

GALLAGA MEP, Arellano RB, Santillán FMA, Favila HLC, Córdova CD, Morales RJ, Díaz AE. 2017. Situación epidemiológica de la paratuberculosis en las principales regiones caprinas de estado de Puebla, México. Quehacer científico en Chiapas. 12(1). 36 - 45. https://dgip.unach.mx/images/pdf-REVISTA-QUEHACERCIENTIFICO/2017-enerjun/4.Situacion_epidemiologica_de_la_paratuberculosis.pdf

JAIMES NG, Santillán MA, Hernández CA, Córdova D, Guzmán CC, Arellano RB, Tenorio GVR, Cuéllar OA. 2008. Detection of Mycobacterium avium subespecie paratuberculosis by nested PCR of ovine fecal samples. Vet Mex. 39(4): 377 - 386. https://www.medigraphic.com/pdfs/vetmex/vm-2008/vm084b.pdf 
JARAMILLO MS, Montoya ZMA, Uribe SJS, Ramírez VNF, Fernández SJA. 2017. Seroprevalencia de paratuberculosis (Mycobacterium avium subsp. paratuberculosis) en un hato de lechería especializada del altiplano norte de Antioquia, Colombia. Revista Veterinaria y Zootecnia 11(2): 24 - 33. http://doi.org/10.17151/vetzo.2017.11.2.3

JARK U, Ringena I, Franz B, Beyerbach M, Gerlach GF. 1997. Development of an ELISA technique for serodiagnosis of bovine paratuberculosis. Vet microbiol. 57: 189 - 198. https://doi.org/10.1016/S0378-1135(97)00125-9

MARTÍNEZ HDI, Sarabia BCDC, Peniche CAEDJ, Villagómez CJA, Magdaleno MA, Hernández RSG, Flores CR. 2012. Seroepidemiology of goat paratuberculosis in five municipalities of Central Veracruz Mexico. Tropical and subtropical agroecosystems, 15 (2): 82-88. https://www.revista.ccba.uady.mx/ojs/index.php/TSA/article/view/1754/769

MEJÍA MK, Lemus FC, González MCA, Palomares RG, Díaz AE, Gutiérrez HJL. 2017. Factores de riesgo asociados a Mycobacterium avium subespecie paratuberculosis en rebaños ovinos de Nayarit, México. Revista Científica FCV - LUZ. 27(5): 294-302. https://www.redalyc.org/jatsRepo/959/95953315005/html/index.html

MILIÁN SF, Santillán FMA, Zendejas MH, García CL, Hernández AL, Cantó AGJ. 2015. Prevalence and associated risk factors for Mycobacterium avium subsp. paratuberculosis in dairy cattle in Mexico. Journal of Veterinary Medicine and Animal Health. 7: 302-307. https://doi.org/10.5897/JVMAH2015.0402

MORALES PMI, Mejía SP, Díaz AE, Palomares REG, Gutiérrez HJL, Reyna GJR, Luna NP, Munguía XJA, Segura CJC, Leyva CJC. 2020. Risk factors associated with the seroprevalence of paratuberculosis in sheep flocks in the hot-arid region of Sonora, México. Tropical Animal Health and Production. 52(3): 1357-1363. http://doi.org/10.1007/s11250-019-02139-y

MORÓN CFJ, Cortéz RC, Gallegos SJ, Figueroa SB, Amante OA. 2013. Prevalencia de la infección por Mycobacterium avium subespecie paratuberculosis en rebaños ovinos de dos municipios de San Luis Potosí, México. Revista Científica FCV - LUZ. 23(4): 293299. ISSN: 0798-2259. https://www.redalyc.org/articulo.oa?id=95926991008

NASER SA, Ghobrial G, Romero C, Valentine JF. 2004. Culture of Mycobacterium avium subspecies paratuberculosis from the blood of patients with Crohn's disease. Lancet. 364:1039-1044. http://doi.org/10.1016/S0140-6736(04)17058-X 
Norma Oficial Mexicana - 1995. NOM-031-Z00-1995. Campaña nacional contra la Tuberculosis bovina Mycobacterium bovis. Secretaria de Agricultura y Desarrollo Rural. México. https://www.gob.mx/senasica/documentos/nom-031-zoo-1995

Norma Oficial Mexicana - 1999. NOM-062-Z00-1999. Especificaciones técnicas para la producción, cuidado y uso de animales de laboratorio. Secretaria de Agricultura y Desarrollo Rural. México.

https://www.gob.mx/cms/uploads/attachment/file/203498/NOM-062-ZOO1999_220801.pdf

OIE. 2020. Organización mundial de sanidad animal: Paratuberculosis. https://www.oie.int/es/sanidad-animal-en-el-mundo/enfermedades-de-losanimales/Paratuberculosis/.

RATHNAIAH G, Zinniel DK, Bannantine JP, Stabel JR, Gröhn YT, Collins MT, Barletta RG. 2017. Pathogenesis, molecular genetics, and genomics of Mycobacterium avium subsp. paratuberculosis, the etiologic agent of Johne's disease. Frontiers in veterinary science. 4:187. http://doi.org/10.3389/fvets.2017.00187

RETAMAL MP, Beltran MC, Abalos PP, Quera PR, Hermoso RM. 2011. Mycobacterium avium subsp paratuberculosis y enfermedad de Crohn: evidencias de una zoonosis. Revista médica Chile. 139(139). ISSN 0034-9887. http://dx.doi.org/10.4067/S003498872011000600015

SEGURA CJ, Honhold N. 2015. Métodos de muestreo para la producción y salud animal. Universidad Autónoma de Yucatán. Mérida, Yucatán, México. Pp. 50 - 52. ISBN 9687556-93-5.

SENASICA. Servicio Nacional de Sanidad, Inocuidad y Calidad Agroalimentaria. 2021. Plan Estratégico conjunto entre Estados Unidos-México para la colaboración de Tuberculosis bovina 2013-2018. México.

https://www.gob.mx/cms/uploads/attachment/file/150563/5_Plan_Estrat_gico_en_Conju nto_SENASICA-APHIS.pdf

SEIOT. Sistema estatal de información para el ordenamiento territorial. 2020. México. http://seiot.sonora.gob.mx/

SIAP. Servicio de Información Agroalimentaria y Pesquero. 2018. Población ganadera (bovinos). México. https://www.gob.mx/siap/documentos/poblacion-ganadera-136762 
VÉLEZ AM, Rendón DY, Valencia RA, Ramírez VN, Fernández SJ. 2016. Seroprevalencia de Mycobacterium avium Subsp. paratuberculosis (MAP) en una granja de ganado de carne de bosque húmedo tropical en Caucasia, Antioquia, Colombia. Revista Colombiana De Ciencia Animal - RECIA. 8(2):167-176. https:/doi.org/10.24188/recia.v8.n2.2016.184

WHITTINGTON R, Donat K, Weber MF, Kelton D, Nielsen SS, Eisenberg S, Arrigoni N, Juste R, Sáez JL, Dhand N, Santi A, Michel A, Barkema H, Kostoulas P, Citer L, Griffin F, Barwell R, Scatamburlo MMA, Slana I, Koehler H, Vir SS, Sang YH, Chávez GG, Goodridge A, Ocepek M, Garrido J, Stevenson K, Collins M, Alonso B, Cirone K, Paolicchi F, Gavey L, Rahman MT, de Marchin E, Praet WV, Bauman C, Fecteau G, McKenna S, Salgado M, Fernández SJ, Dziedzinka R, Echeverria G, Seppänen, Thibault V, Fridriksdottir V, Derakhshandeh A, Haghkhah M, Ruocco L, Kawaii S, Momotani E, Heuer C, Norton S, Cadmus S, Agdestein A, Kampen A, Szteyn J, Frössling J, Schwan E, Caldow G, Strain S, Carter M, Wells S, Munyeme M, Wolf R, Gurung R, Verdugo C, Fourichon C, Yamamoto T, Thapaliva S, Di Labio E, Ekgatat M, Gil A, Nuñez AA, Piaggio J, Suanes A, de Warrd JH. 2019. Control of paratuberculosis: who, why and how. A review of 48 countries. BMC Vet Res. 15:198. https://doi.org/10.1186/s12917-019-1943-4

ZAREI KF, Geramizadeh B, Khodakaram T A. 2019. Prevalence of Mycobacterium avium subspecies paratuberculosis IS 900 DNA in biopsy tissues from patients with Crohn's disease: histopathological and molecular comparison with Johne's disease in Fars province of Iran. BMC infectious diseases. 19(1):23. https://doi.org/10.1186/s12879-018$3619-2$ 